Chronic Obstructive Pulmonary Diseases: Journal of the COPD Foundation

\author{
Original Research
}

\title{
A Retrospective Claims Analysis of Dual Bronchodilator Fixed- Dose Combination Versus Bronchodilator Monotherapy in Patients with Chronic Obstructive Pulmonary Disease
}

Charlie Strange, $\mathrm{MD}^{1}$ Valery Walker, $\mathrm{MS}^{2}$ Junliang Tong, $\mathrm{PhD}^{2}$ Jonathan Kurlander, $\mathrm{MS}^{2}$ Maureen Carlyle, $\mathrm{MPH}^{2}$ Lauren A. Millette, $\mathrm{PhD}^{3}$ Eric Wittbrodt, PharmD, $\mathrm{MPH}^{3}$

\begin{abstract}
Introduction: Patients with chronic obstructive pulmonary disease (COPD) increasingly receive combination bronchodilator therapies. Real world evidence for the benefits of combination therapy compared to monotherapy is lacking.
\end{abstract}

Methods: COPD patients aged $\geq 40$ years initiating monotherapy (MT) with either a long-acting muscarinic antagonist (LAMA) or long-acting beta2-agonist (LABA) or dual therapy (DT) with a LAMA/LABA fixed dose combination (FDC) between January 1, 2016 and December 31, 2016 were identified from a large U.S. administrative claims database. Patients diagnosed with cystic fibrosis, idiopathic pulmonary fibrosis, or asthma were excluded. Cohorts were propensity score matched 1:1 using baseline measures (e.g., exacerbations, hospitalizations) as proxies for COPD severity to create balanced cohorts.

Results: Following propensity score matching (PSM), 1286 patients remained in each cohort for analysis. Patients were followed for approximately 1 year. Patients in the DT versus MT cohort had lower rates of exacerbations leading to hospitalization (incidence rate ratio 0.7886; $p=0.019$ ), lower mean COPD-related pharmacy costs per patient per month (PPPM) ( $\$ 300$ versus $\$ 379$, respectively; $p<0.001)$ and total costs PPPM ( $\$ 990$ versus $\$ 1203$, respectively; $p=0.003)$. This occurred despite lower mean COPD-related pharmacy fills PPPM in the DT versus MT cohorts (1.41 versus 1.51, respectively; $p=0.038$ ). Patients in the DT cohort had lower rates of switching $(p<0.001)$ and augmentation $(p<0.001)$, and higher rates of non-persistence $(p<0.001)$ versus the MT cohort. Rates of discontinuation were similar.

Conclusions: Patients in the DT cohort had lower rates of exacerbations leading to hospitalization, lower COPDrelated pharmacy and total costs PPPM, and lower rates of switching and augmentation compared to patients in the MT cohort.

\footnotetext{
Abbreviations: chronic obstructive pulmonary disease, COPD; monotherapy, MT; long-acting muscarinic antagonist, LAMA; long-acting beta2-agonist, LABA; dual therapy, DT; fixed-dose combination, FDC; propensity score matching, PSM; per patient per month, PPPM; Global initiative for chronic Obstructive Lung Disease, GOLD; inhaled corticosteroid, ICS; Medicare Advantage with Part D, MAPD; International Classification of Diseases, Ninth Revision, Clinical Modification, ICD-9-CM; emergency department, ED; medication possession ratio, MPR; proportion of days covered, PDC; short-acting muscarinic antagonist, SAMA; short-acting beta2-agonist, SABA; Agency for Healthcare Research and Quality, AHRQ; incidence rate ratio, IRR; standard deviation, SD

Funding Support: This study was funded by AstraZeneca Pharmaceuticals LP (Wilmington, Delaware).

Date of Acceptance: April 5, 2019

Citation: Strange C, Walker V, Tong J, et al. A retrospective claims analysis of dual bronchodilator fixed-dose combination versus bronchodilator monotherapy in patients with chronic obstructive pulmonary disease. Chronic Obstr Pulm Dis. 2019;6(3):221-232. doi: https://doi.org/10.15326/jcopdf.6.3.2018.0160
} 
1 Division of Pulmonary and Critical Care Medicine, Medical University of South Carolina, Charleston

2 Optum, Inc., Eden Prairie, Minnesota

3 AstraZeneca, Wilmington, Delaware

\section{Address correspondence to:}

Valery Walker

Optum, Inc.

11000 Optum Circle

Eden Prairie, MN 55344

Email: Valery.walker@optum.com

\section{Keywords:}

fixed-dose combination; long-acting muscarinic antagonist; LAMA; long-acting beta2-agonist; LABA; LAMA/LABA; administrative claims, retrospective analysis; chronic obstructive pulmonary disease; $\mathrm{COPD}$

\section{Introduction}

Chronic obstructive pulmonary disease (COPD) ranks fourth after cancer, heart disease, and unintentional injuries as a leading cause of death in the United States. ${ }^{1}$ Patients with COPD typically experience persistent respiratory symptoms and airflow obstruction. ${ }^{2}$ The total medical costs associated with COPD in 2010 in the United States were estimated at $\$ 32.1$ billion, with costs due to work absenteeism accounting for an additional $\$ 3.9$ billion. $^{3}$

For the treatment of moderate or severe COPD, the Global initiative for chronic Obstructive Lung Disease $(G O L D)^{2}$ recommends either monotherapy (MT) with a long-acting muscarinic antagonist (LAMA) or a long-acting beta2-agonist (LABA), or combination therapy with a LAMA/LABA, inhaled corticosteroid (ICS)/LABA, or ICS/LAMA/LABA. Decisions among these medication classes are stratified by severity of symptoms and exacerbation frequency and severity, instead of spirometric category (level of forced expiratory volume in 1 second [FEV 1$]$ ).

A retrospective administrative claims study conducted by Dalal and colleagues ${ }^{4}$ reported that the frequency of exacerbations in the first year of the study (2008) predicted the frequency and intensity of exacerbations in the subsequent 2 -year period. They also reported significantly higher COPD-related mean annual per-patient costs for patients who experienced frequent (i.e., $\geq 2$ ) exacerbations in the first year $(\$ 3565$ in 2009 and $\$ 3528$ in 2010), compared to those who did not experience exacerbations in the first year ( $\$ 1007$ in 2009 and $\$ 1027$ in 2010; $P<0.05$ ). Although Dalal et al reported that ICS/LABA was the most common type of combination therapy used across exacerbation categories, they did not report further detail such as the number of patients receiving other combination therapies.

Patients with COPD increasingly receive combination bronchodilator therapies. Evidence examining whether the addition of a second bronchodilator improves patient outcomes in a realworld setting is lacking. The objective of this study was to compare the frequency of COPD-related exacerbations, all-cause and COPD-related health care resource utilization and costs, between patients with COPD initiating LAMA/LABA fixed-dose combination (FDC) therapy (DT) versus LAMA or LABA MT, adjusted for baseline characteristics as proxies for COPD severity.

\section{Methods}

\section{Study Design}

This observational retrospective analysis used the Optum Research Database, which includes approximately 14 million enrollees in commercial U.S. health insurance plans and 3 million enrollees in Medicare Advantage with Part D (MAPD) plans annually. This medical and pharmacy administrative claims database was used to identify patients with treatment initiation (index date) of a LAMA, LABA, or LAMA/LABA FDC between January 1, 2016 to December 31, 2016. The follow-up period was variable from January 1, 2016, to June 30, 2017, depending on the index date; however, all patients were followed for $\geq 6$ months. The baseline period was the 9 -month period before treatment initiation (Figure 1).

A subset of patients identified for this study was invited to participate in a separate survey study. ${ }^{5}$ The retrospective analysis reported here was included in the survey protocol, which received ethics approval and a waiver of authorization from the New England Institutional Review Board.

\section{Population}

Inclusion Criteria: Patients were included if they were aged $\geq 40$ years as of the year of the index date and had $\geq 1$ pharmacy claim for MT with either a LAMA or LABA or FDC DT with LAMA/LABA (see online data supplement, eTable 1) during the identification period (index date=first prescription date). In addition, they 


\section{Figure 1. Identification and Observation Periods}

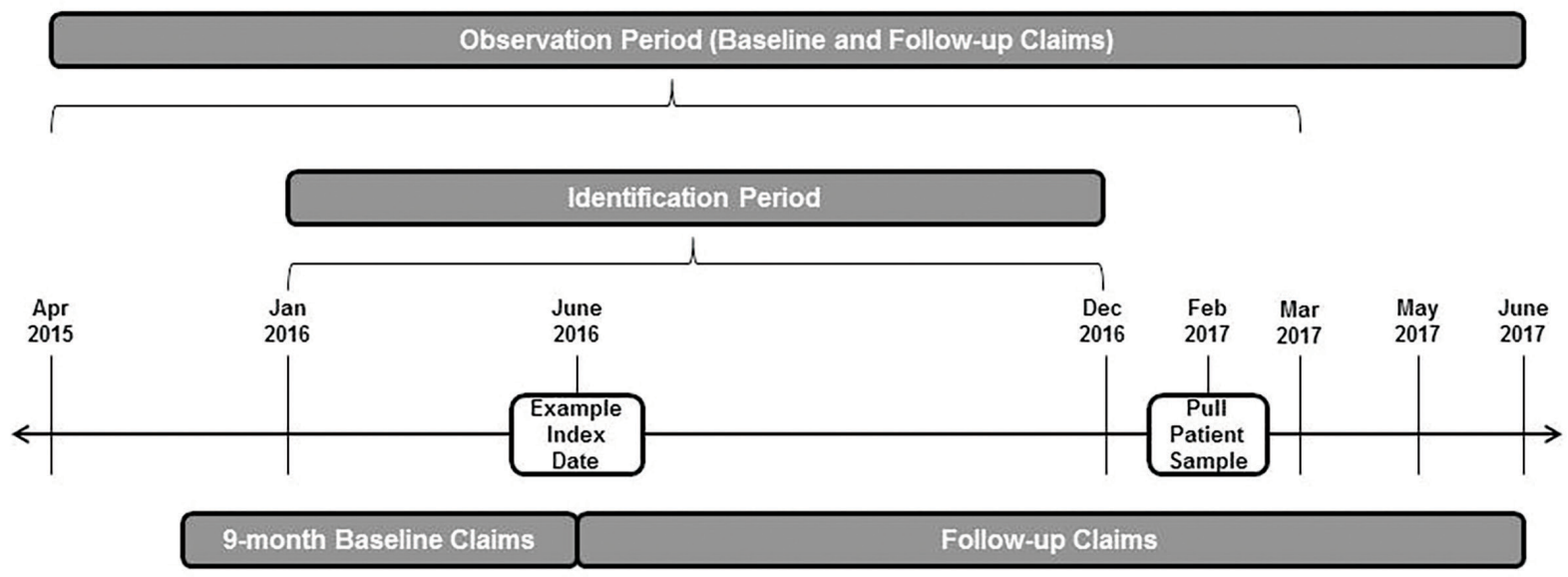

were required to have $\geq 2$ medical claims $\geq 30$ days apart with an International Classifications of Diseases, Ninth Revision, Clinical Modification (ICD-9-CM) or ICD-Tenth Revision-CM (ICD-10-CM) diagnosis code for COPD (see online data supplement eTable 2) in any position (to limit the likelihood of capturing rule-out testing, diagnostic claims were excluded); continuous enrollment in the health plan from the index date to June 2017 (follow-up period) and 9 months prior to the index date (baseline period); and any day supply of index medication on hand within the month prior to patient identification for survey invitation.

Exclusion Criteria: Patients were excluded if they were diagnosed with cystic fibrosis, idiopathic pulmonary fibrosis, or asthma (See online data supplement, eTable 3) during the identification or baseline period; $\geq 1$ pharmacy claim for LAMA, LABA, or LAMA/ LABA FDC within 3 months prior to index date; $\geq 3$ pharmacy claims for any of these medications within 9 months prior to the index date; or $\geq 1$ pharmacy claim for an ICS or ICS/LABA within 2 months prior to the index date.

\section{Cohorts}

The DT cohort consisted of patients initiating a LAMA/ LABA FDC with no prior LAMA, LABA, or LAMA/ LABA FDC in the 3 months prior to the index date and $<3$ prescription fills of any of these medications in the 9 months prior to the index date, and no ICS or ICS/ LABA in the 2 months prior to the index date.

The MT cohort consisted of patients initiating a LAMA or LABA with no prior LAMA, LABA, or LAMA/ LABA FDC in the 3 months prior to the index date and
$<3$ prescription fills of any of these medications in the 9 months prior to the index date, and no ICS or ICS/ LABA in the 2 months prior to the index date.

\section{Outcomes}

COPD Exacerbations: COPD exacerbations included those scenarios in which each episode could consist of multiple health care encounters (exacerbation events), and in which the exacerbation ended when 14 days passed without any exacerbation events. COPD exacerbation events were defined as an inpatient hospitalization with a primary diagnosis for COPD (start and end dates of exacerbation were defined as the inpatient hospitalization admit and discharge dates, respectively), or an emergency department (ED) visit with a diagnosis in any position for COPD (start and end dates of exacerbation were defined as the ED service date). Also included as an exacerbation event was an ambulatory visit (office or outpatient) with a diagnosis in any position for COPD and a procedure code for steroid and/or antibiotic administration during the visit, or a pharmacy claim for a steroid and/or antibiotic on the same day or within 10 days following the ambulatory visit (start and end dates of the exacerbation were defined as the ambulatory visit service date).

COPD Severity: Each exacerbation episode was classified as severe or moderate based on the site of care. COPD-related hospitalizations or COPD-related ED visits were classified as severe. COPD-related ambulatory visits were classified as moderate.

Medication Use: Medication possession ratio (MPR) was used as a measure of compliance with the index 
therapy. MPR was calculated by summing the number of days supplied of the index therapy for all but the last fill in the observation period and dividing the sum by the number of days between the first and the last prescription refill.

Proportion of days covered (PDC) was also used as a measure of compliance and represents the proportion of time over the course of a patient's index therapy that he/she theoretically was in possession of the medication. PDC was calculated by dividing the number of days on which a medication was available (based on filled prescriptions) by the number of days between the earliest prescription claim in the observation period through the end of the observation period.

A change in index therapy was measured for the entire follow-up period; however, patients who changed therapy could only be classified into 1 of the following categories: discontinuation (defined as a gap in therapy of $\geq 60$ days), switch, augmentation, or step down. Also, any changes following the initial change were not captured. A switch in therapy was defined as a discontinuation of the index therapy and start of a new therapy within 60 days after the index therapy run-out date. Augmentation was defined as additional therapy added to the index therapy within 60 days after the index therapy run-out date. Step down was defined as removal of a therapy in the index therapy within 60 days after the index therapy run-out date. Two additional medication measures in addition to the main 4 measures included discontinuation of index therapy defined as a gap in therapy of $\geq 90$ days and non-persistence defined as at least 1 interrupted day on therapy.

Health Care Utilization and Costs: All-cause and COPD-related health care utilization and costs were calculated for the baseline and follow-up periods. "Allcause" included all health care utilization consumed during the study regardless of the diagnosis, and "COPD-related" included medical claims with a COPD diagnosis (see online data supplement, eTable 2) in any position, or if the pharmacy claim was for a COPD medication (see online data supplement, eTable 4). Also included were claims that did not have a COPD diagnosis but occurred between the start and end dates of an exacerbation +14 days or was a diagnostic workup procedure (e.g., pulmonary imaging, spirometry) or breathing assistance (e.g., oxygen, ventilation). The diagnostic work-up procedures and breathing assistance were reported as a separate category, "tests and procedures," for COPD-related costs. Costs combined health plan and patient-paid amounts and were adjusted using the annual medical care component of the Consumer Price Index. ${ }^{6}$

\section{Statistical Analysis}

Because the study cohorts were non-randomized, propensity score matching $(\mathrm{PSM})^{7}$ was performed to balance or adjust for confounders between study cohorts. Patients were matched to one another at a 1:1 ratio to create study cohorts with balanced baseline characteristics. To create the propensity score, a logistic regression model was fitted for LAMA or LABA monotherapy versus LAMA/LABA FDC use as a function of the patients' demographic, clinical characteristics, as well as baseline claims measures. These measures were used as proxies for COPD severity (see online data supplement, eTable 5). These model covariates were available up to the index date and thus had the potential to influence assignment of the index drug.

The baseline measures that were used as proxies for COPD severity included patient out-of-pocket health care costs, total health care costs, number of ED visits, age, gender, Quan-Charlson comorbidity score, ${ }^{8}$ and baseline use of a nebulizer.

Also included as proxies for COPD severity were 10 of the 12 components from Wu et al's (2006) claims-based COPD severity score ${ }^{9}$ (see online data supplement, eTable 5). An actual severity score based on Wu et al was not calculated because 12 months of claims were required, and the present study included only 9 months. Anti-cholinergic and long-acting bronchodilators were removed because they were the index medications in this study, and short-acting muscarinic antagonists (SAMAs) were added because baseline counts of short-acting beta2-agonists (SABAs) were included as one of Wu's 12 components. The following were included: hospitalization due to acute exacerbation of chronic bronchitis, oxygen therapy, emphysema, spirometry tests, pulmonologist visits, oral corticosteroids, inhaled corticosteroids, SABAs, SAMAs, and age (see online data supplement, eTable 5).

Because patients had a variable length of follow-up, results such as counts for exacerbations, counts for health care resource utilization, and mean costs, were reported as per patient per month (PPPM). 
Exacerbations and index medication treatment patterns (e.g., discontinuation) were reported as incidence rate ratios (DT divided by $\mathrm{MT}$ ), and comparisons were tested using an exact binomial test. A negative binomial regression model of incidence with an offset of follow-up days was used to compare the incidence rate of exacerbations between the 2 cohorts, controlling for baseline covariates. The baseline covariates used in the models were the same covariates used in the PSM. Even though the covariates were used in the PSM, they were also used again in the models to customize the relationship to that specific model outcome.

A logistic regression model was used to compare the incidence rates of COPD-related hospitalizations between the 2 cohorts, controlling for baseline covariates. A gamma regression model with log link was used for the comparison of COPD-related costs and the all-cause costs between the 2 cohorts, while controlling for baseline covariates. Chi-square tests and t-tests were used for comparisons of demographic and outcome measures. Significance tests were 2 -tailed and carried out at a .05 level of significance.

\section{Results}

\section{Baseline Findings}

Following PSM, 1286 patients remained in each cohort for the analysis (MT: $n=1238$ taking a LAMA, $n=48$ taking a LABA). The 2 cohorts were similar in mean age (MT $69.8 \pm 9.4$ versus DT $69.7 \pm 9.7$ years) and gender (MT 50.9\% versus DT 49.5\% female) (Table 1). Approximately three-quarters of patients in both cohorts were MAPD health plan enrollees, and 93\% lived in an urban area. A greater percentage of patients in the DT than MT cohort had a pulmonologist prescribe their index medication. In contrast, a greater percentage of patients in the MT cohort had a general practitioner or internist prescribe their index medication compared to the patients in the DT cohort.

The 2 cohorts had similar Quan-Charlson comorbidity mean scores (MT 2.16 versus DT 2.26; $P=0.154$ ), and similar percentages of patients with the top 10 Agency for Healthcare Research and Quality (AHRQ) comorbid conditions. Exceptions included a higher percentage of patients in the DT cohort with symptoms related to lower respiratory disease and disorders of lipid metabolism, compared to the MT cohort.
The 2 cohorts had similar baseline mean COPDrelated exacerbations PPPM, including those leading to hospitalization, those leading to ED visits, and those classified as severe or moderate. A similar percentage of patients were taking a SAMA or a SABA, but a greater percentage of patients in the DT versus $M T$ cohort were taking a SAMA/SABA combination rescue medication.

The 2 cohorts had similar baseline all-cause and COPD-related costs PPPM, and similar all-cause and COPD-related health care resource utilization (Table 1). Exceptions included a higher percentage of patients with a COPD-related office visit (DT 89.0\% versus MT $81.1 \%$, respectively; $P<0.001)$ as well as COPD-related tests and procedures (DT $82.7 \%$ versus MT $79.5 \%$, $P=0.034)$, and a higher mean count of COPD-related office visits PPPM (DT 0.40 versus MT 0.35, $\mathrm{P}=0.001$ ) and all-cause office visits PPPM (DT 1.48 versus MT 1.37, $P=0.020)$ for the DT than MT cohort.

\section{Follow-up Findings}

The mean duration of follow-up was significantly longer for the MT versus the DT cohort (mean 373.69 days versus 326.54 days; $P<0.001$ ). Rescue medication use was similar for the 2 cohorts, as reflected in the mean average days of supply PPPM of SAMA (DT 6.30 versus MT 6.10 days; $P=0.903$ ), SABA (DT 8.06 versus MT 8.11; $P=0.883$ ) and SAMA/SABA (DT 9.18 versus $\mathrm{MT}$ 7.82; $P=0.080$ ), and the mean average number of prescription fills PPPM of SAMA (DT 0.19 versus MT 0.19; $P=0.946$ ), SABA (DT 0.31 versus $M T$ $0.30 ; P=0.445$ ) and SAMA/SABA (DT 0.32 versus MT $0.29 ; P=0.395)$. Index medication use was similar as measured by MPR (DT 77\% versus MT 76\%; $P=0.242$ ), but PDC was significantly lower in the DT (0.58) versus the MT (0.63) cohort $(P<0.001)$.

The DT cohort had lower rates of switching than the MT cohort (incidence rate ratio [IRR]: $0.19, P<0.001$ ) and augmentation (IRR: 0.02, $P<0.001$; Table 2). Fortytwo patients in the DT cohort switched from LAMA/ LABA FDC to ICS/LABA FDC, and 154 patients in the MT cohort switched from LAMA to ICS/LABA FDC. Nine patients in the DT cohort and 358 patients in the MT cohort augmented to triple therapy. The DT cohort had higher rates of non-persistence compared to the MT cohort (IRR: 1.34, $P<0.001$ ) (Table 2). There were similar rates of discontinuation for the DT versus the MT cohort (IRR: $1.02 \geq 60$ days gap, $P=0.714$; IRR: 1.12, $\geq 90$ days gap, $P=0.116$; Table 2 ). 


\section{Table 1. Baseline Cohort Characteristics}

Characteristic

Characteristic
LAMA or

LABA MT

$(\mathrm{N}=1286)$

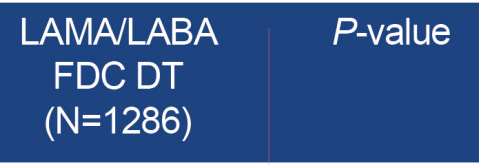

$69.75(9.44)$

655 (50.93)

631 (49.07)

\begin{tabular}{|r|}
\hline $213(16.56)$ \\
\hline $432(33.59)$ \\
\hline $542(42.15)$ \\
\hline $99(7.70)$
\end{tabular}

304 (23.64)

$982(76.36)$

Medicare Advantage

U.S. Census Bureau Core Based Statistical Area, ${ }^{a} \mathrm{n}(\%)$

Geographic Region, n (\%)

Northeast

$69.67(9.74)$

0.842

$649(50.47)$

0.478

\begin{tabular}{|r|r|}
\hline $161(12.52)$ & 0.004 \\
\hline $310(24.11)$ & $<0.001$ \\
\hline $713(55.44)$ & $<0.001$ \\
\hline $102(7.93)$ & 0.826 \\
\hline & \\
\hline $336(26.13)$ & 0.144 \\
\hline $950(73.87)$ & \\
\hline
\end{tabular}

1199 (93.23)

$87(6.77)$

Rural

(\%)

Pulmonologist

Allergist

General Practitioner/Internist

Other Specialties

394 (38.93)

$14(1.38)$

$531(52.47)$

$73(7.21)$

Charlson Comorbidity Score, mean (SD)

Top 10 AHRQ Comorbid Conditions, ${ }^{\mathbf{b}} \mathrm{n}(\%)$

COPD and Bronchiectasis

$2.16(1.71)$

Hypertension

1201 (93.39)

930 (72.32)

Other Lower Respiratory Disease

$913(71.00)$

Disorders of Lipid Metabolism

Diseases of the Heart

Diseases of the Urinary System

Non-traumatic Joint Disorders

Respiratory Infections

Other Connective Tissue Disease

$791(61.51)$

$791(61.51)$

499 (38.80)

$472(36.70)$

495 (38.49)

Spondylosis; InterVertebral Disc Disorders; Other Back Problems

477 (37.09)

$470(36.55)$

Number of COPD Exacerbations PPPM, mean (SD)

Leading to Hospitalization

Leading to ED or Urgent Care Facility Visit

Severe

Moderate

$0.09(0.13)$

$0.03(0.06)$

$0.05(0.09)$

$0.01(0.05)$

$0.08(0.12)$

Rescue Medication Use, $\mathrm{n}(\%)$

SAMA Use

SABA Use

$32(2.49)$

SAMA/SABA Use

659 (51.24)

150 (11.66)

All-Cause Resource Utilization, $\mathrm{n}(\%)$

Office Visit

$1248(97.05)$

1018 (79.16)

Outpatient Visit

\begin{tabular}{|r|r|}
\hline $488(47.29)$ & $<0.001$ \\
\hline $5(0.48)$ & 0.034 \\
\hline $475(46.03)$ & 0.004 \\
$64(6.20)$ & 0.360 \\
\hline $2.26(1.80)$ & 0.154 \\
\hline
\end{tabular}

1217 (94.63) $\quad 0.184$

$\begin{array}{ll}957(74.42) & 0.228\end{array}$

$979(76.13) \quad 0.003$

$843(65.55) \quad 0.033$

$786(61.12) \quad 0.840$

$516(40.12) \quad 0.493$

$478(37.17) \quad 0.806$

$510(39.66) \quad 0.544$

$481(37.40) \quad 0.870$

$467(36.31) \quad 0.902$

$0.09(0.12) \quad 0.701$

$0.02(0.05) \quad 0.217$

$0.05(0.09) \quad 0.212$

$0.01(0.04) \quad 0.417$

$0.08(0.12) \quad 0.470$


ED Visit

520 (40.44)

$512(39.81)$

0.748

Inpatient Stay

$293(22.78)$

$298(23.17)$

0.815

Pharmacy Use

$1272(98.91)$

1280 (99.53)

0.073

COPD-Related Resource Utilization, $\mathrm{n}(\%)$

\begin{tabular}{|l|r|r|r}
\hline Office Visit & $1043(81.10)$ & $1145(89.04)$ & $<0.001$ \\
\hline Outpatient Visit & $609(47.36)$ & $642(49.92)$ & 0.193 \\
\hline ED Visit & $321(24.96)$ & $309(24.03)$ & 0.582 \\
\hline Inpatient Stay & $253(19.67)$ & $258(20.06)$ & 0.805 \\
\hline Pharmacy Use & $1083(84.21)$ & $1056(82.12)$ & 0.155 \\
\hline Tests and Procedures & $1022(79.47)$ & $1064(82.74)$ & 0.034 \\
\hline Health Care Costs (\$) PPPM, mean (SD) & & & \\
\hline All-Cause & $1618.44(2886.27)$ & $1675.00(2860.39)$ & 0.618 \\
\hline COPD-Related & $731.01(1920.38)$ & $759.64(1927.34)$ & 0.706 \\
\hline
\end{tabular}

LAMA=long-acting muscarinic antagonist; LABA=long-acting beta2-agonist; MT=monotherapy; FDC=fixed-dose; combination; $\mathrm{DT}=$ dual therapy; $\mathrm{AHRQ}=$ Agency for Healthcare Research and Quality; COPD=chronic obstructive pulmonary disease;

PPPM=per patient per month; $\mathrm{SD}=$ standard deviation; $\mathrm{ED}=$ emergency department; SAMA=short-acting muscarinic antagonist; $\mathrm{SABA}=$ short-acting beta2-agonist

${ }^{a}$ Based on the U.S. Census Bureau Core Based Statistical Area.

${ }^{\mathrm{b}}$ Comorbidities defined by Healthcare Cost and Utilization Project (HCUP) comorbidity software. ${ }^{10}$ The AHRQ CCS requires at least 1 medical claim to flag each condition.

Exacerbations: The mean number of exacerbations PPPM was similar in both cohorts as were the mean number of severe and moderate exacerbations PPPM (Table 3). The negative binomial regression model for the incidence rate for exacerbations adjusted for baseline covariates found no differences between cohorts (IRR: 0.96, $P=0.345$ ). The DT cohort had a lower incidence rate of exacerbations leading to hospitalization when compared to the MT cohort (0.14 versus 0.18 , IRR: $0.79, P=0.019$; Table 4). There were no significant cohort differences in the rates of exacerbations leading to an ED or office visit.

Health Care Resource Utilization and Costs: The cohorts were similar in all-cause health care resource utilization and costs, and COPD-related resources, with the exception of fewer pharmacy fills PPPM in the DT versus $\mathrm{MT}$ cohort (1.41 versus $1.51 ; P=0.038$ ) (Table 5). The DT versus MT cohort had lower mean COPD-related pharmacy cost PPPM (\$300 versus $\$ 379 ; P<0.001)$ and total costs PPPM (\$990 versus $\$ 1203 ; P=0.003$; Table 5).

In the gamma regression models adjusted for baseline covariates, DT had lower all-cause total costs PPPM (cost ratio: $0.91, P=0.036$ ) and lower COPDrelated total costs PPPM (cost ratio: 0.79, $P<0.001$ ) compared to the $\mathrm{MT}$ cohort. In the logistic regression model adjusted for baseline covariates, the DT cohort had fewer COPD-related hospitalizations (odds ratio: 0. 62, $P<0.001)$ compared to the MT cohort.

\section{Discussion}

Appropriate use of bronchodilator medication in COPD treatment remains a topic of debate. The addition of a second class of bronchodilator to maximal doses of 1 bronchodilator adds numerically small increments to the peak post-bronchodilator $\mathrm{FEV}_{1}$ response. ${ }^{11}$ However, when other COPD outcomes are measured, the second class of bronchodilator has been shown to reduce exacerbation frequency ${ }^{12}$ and dyspnea ${ }^{13}$ and to improve exercise endurance ${ }^{14}$ compared to monotherapy. Therefore, a new class of LAMA/LABA FDC medications were developed and studied to determine if the added convenience of 2 medications in a single inhaler would translate to meaningful health benefits. Only now can real-world use of this new class of medication be studied in COPD.

Real-world studies often produce different results than prospective, randomized controlled trials. There are multiple reasons for this discrepancy; among them is the fact that research study participants are heavily screened to obtain a population likely to achieve optimal treatment outcomes. Drug utilization is monitored and compliance is stressed. Study participants are encouraged to stay on allocated treatment assignments to obtain meaningful study outcomes that inform the science of COPD treatment response. Importantly, study medications are provided 
without cost.

In contrast, real-world studies recognize that medication adherence is dismal in most COPD patients. The refill rates of controller medications average $40 \%-70 \%,{ }^{15,16}$ and a major target of COPD disease management programs is to improve drug utilization known to decrease exacerbation frequency. ${ }^{17}$

One goal of LAMA/LABA FDC medications is to maximally improve symptoms that might improve downstream compliance. The problem with the study of index medication use in COPD is that many patients likely initiate therapy in the context of a physician visit for an exacerbation. The stimulus to continue medication after the exacerbation has resolved is likely dependent on disease severity. Therefore, this study design using propensity matching was the best attempt to include some minimally symptomatic early COPD patients and some more severe COPD patients within the same study cohort.

In this context, the findings of the current study suggest that patients in the DT cohort had better COPD control versus patients in the MT cohort as

\section{Table 2. Changes in Index Medication During Follow-up}

\begin{tabular}{|c|c|c|c|c|c|c|c|c|}
\hline \multirow[b]{2}{*}{ Medication Change } & \multicolumn{3}{|c|}{$\begin{array}{l}\text { LAMA or LABA MT } \\
\qquad(N=1286)\end{array}$} & \multicolumn{3}{|c|}{$\begin{array}{l}\text { LAMAVLABA FDC DT } \\
(\mathrm{N}=1286)\end{array}$} & \multicolumn{2}{|c|}{$\begin{array}{l}\text { LAMA/LABA } \\
\text { FDC DT vs } \\
\text { LAMA or } \\
\text { LABA MT }\end{array}$} \\
\hline & Events & $\begin{array}{l}\text { Person } \\
\text { Years }\end{array}$ & $\begin{array}{l}\text { ncidence } \\
\text { Rate }\end{array}$ & Events & $\begin{array}{l}\text { Person } \\
\text { Years }\end{array}$ & $\begin{array}{l}\text { ncidence } \\
\text { Rate }\end{array}$ & $\begin{array}{l}\text { Incidence } \\
\text { Rate Ratio }\end{array}$ & $P$-value \\
\hline \multicolumn{9}{|l|}{ Discontinuation } \\
\hline$\geq 60$ days gap & 392 & 577 & 0.6793 & 559 & 803 & 0.6960 & 1.0246 & 0.714 \\
\hline$\geq 90$ days gap & 296 & 637 & 0.4647 & 454 & 869 & 0.5225 & 1.1243 & 0.116 \\
\hline Non-Persistence & 1180 & 373 & 3.1640 & 1217 & 287 & 4.2348 & 1.3384 & $<0.001$ \\
\hline Switch & 163 & 577 & 0.2825 & 42 & 803 & 0.0523 & 0.1851 & $<0.001$ \\
\hline LABA MT to LAMA MT & 3 & 577 & 0.0052 & & & & & \\
\hline LAMA MT to LABA MT & 6 & 577 & 0.0104 & & & & & \\
\hline LAMA MT to ICS/LABA FDC DT & 154 & 577 & 0.2669 & & & & & \\
\hline LABA/LAMA FDC DT to ICS/LABA FDC DT & & & & 42 & 803 & 0.0523 & & \\
\hline Augmentation & 383 & 577 & 0.6637 & 9 & 803 & 0.0112 & 0.0169 & $<0.001$ \\
\hline LABA MT to LABA + LAMA DT & 1 & 577 & 0.0017 & & & & & \\
\hline LAMA MT to LAMA + LABA DT & 1 & 577 & 0.0017 & & & & & \\
\hline LAMA MT to LABA/LAMA FDC DT & 23 & 577 & 0.0399 & & & & & \\
\hline LABA MT to ICS/LABA FDC+LAMA TT & 1 & 577 & 0.0017 & & & & & \\
\hline LAMA MT to ICS/LABA FDC+LAMA TT & 357 & 577 & 0.6187 & & & & & \\
\hline $\begin{array}{l}\text { LABA/LAMA FDC DT to ICS/ } \\
\text { LABA FDC+LAMA TT }\end{array}$ & & & & 9 & 803 & 0.0112 & & \\
\hline \multicolumn{9}{|l|}{ Step Down } \\
\hline LABA/LAMA FDC DT to LABA MT & & & & 1 & 803 & 0.0012 & & \\
\hline LABA/LAMA FDC DT to LAMA MT & & & & 7 & 803 & 0.0087 & & \\
\hline
\end{tabular}

LAMA=long-acting muscarinic antagonist; LABA=long-acting beta2 agonist; MT=monotherapy; FDC=fixed-dose combination; DT=dual therapy; ICS=inhaled corticosteroids; TT=triple therapy

Discontinuation of therapy was defined as a gap in therapy of $\geq 60$ days or $\geq 90$ days.

Non-persistence was defined as at least 1 interrupted day on therapy.

Discontinuation of index therapy was defined as a gap in therapy of $\geq 60$ days.

Switch was defined as a discontinuation of the index therapy and start of a new therapy within 60 days after the index therapy run-out date.

Augmentation was defined as additional therapy added to the index therapy within 60 days after the index therapy run-out date.

Step down was defined as removal of a therapy in the index therapy within 60 days after the index therapy run-out date.

Person-years begins at index date to the event date or end of follow-up.

Incidence Rate is the number of events divided by person-years.

Incidence Rate Ratio is the rate for DT divided by the rate of MT. 


\section{Table 3. Number of Exacerbations per Patient per Month During Follow-up}

\begin{tabular}{l|c|c|c} 
& $\begin{array}{c}\text { LAMA or LABA MT } \\
(\mathrm{N}=1286)\end{array}$ & $\begin{array}{c}\text { LAMALABA } \\
\text { FDC DT } \\
(\mathrm{N}=1286)\end{array}$ & $P$-value \\
\hline Number of COPD Exacerbations PPPM, mean (SD) & $0.11(0.14)$ & $0.11(0.14)$ & 0.391 \\
\hline Severe & $0.02(0.05)$ & $0.01(0.04)$ & 0.067 \\
\hline Moderate & $0.10(0.12)$ & $0.10(0.13)$ & 0.793 \\
\hline
\end{tabular}

LAMA=long-acting muscarinic antagonist; LABA=long-acting beta2-agonist; MT=monotherapy; FDC=fixed-dose combination;

$\mathrm{DT}=\mathrm{dual}$ therapy; $\mathrm{COPD}=$ chronic obstructive pulmonary disease; $\mathrm{PPPM}=$ per patient per month; $\mathrm{SD}=\mathrm{standard}$ deviation

\section{Table 4. Exacerbation Incidence Rates During Follow-up}

\section{LAMA or LABA MT LAMA/LABA FDC DT $(\mathrm{N}=1286)$ \\ $(\mathrm{N}=1286)$}

\section{LAMA/LABA FDC DT vs \\ LAMA or \\ LABA MT}

Type of Exacerbation

Events Person-Incidence Events Person-Incidence Incidence $P$-value Years Rate Years

Rate Rate Ratio

\begin{tabular}{|l|l|l|l|l|l|l|l|l|}
\hline Leading to Hospitalization & 238 & 1317 & 0.1808 & 164 & 1151 & 0.1425 & 0.7886 & 0.019 \\
\hline $\begin{array}{l}\text { Leading to ED or Urgent Care Facility } \\
\text { Visit }\end{array}$ & 445 & 1317 & 0.3380 & 348 & 1151 & 0.3025 & 0.8949 & 0.121 \\
\hline $\begin{array}{l}\text { Leading to an Office Visit(s) and a } \\
\text { Steroid and/or Antibiotic within 10 } \\
\text { Days }\end{array}$ & 1039 & 1317 & 0.7891 & 928 & 1151 & 0.8066 & 1.0221 & 0.628 \\
\hline
\end{tabular}

LAMA=long-acting muscarinic antagonist; LABA=long-acting beta2 agonist; MT=monotherapy; FDC=fixed-dose combination; $\mathrm{DT}=$ dual therapy; ED=emergency department

Person-years begins at index date to the event date or end of follow-up.

Incidence Rate is the number of events divided by person-years.

Incidence Rate Ratio is the rate for DT divided by the rate of MT.

evidenced by less switching and augmentation, fewer exacerbations leading to hospitalization, and lower COPD-related pharmacy and total costs. Minimal use of LABA MT observed in the current study reflects prescribing patterns in contemporary clinical practice.

Our finding that the DT cohort experienced fewer exacerbations leading to hospitalization when compared to the MT cohort is similar to findings from a study conducted by Wedzicha (2013), ${ }^{18}$ who compared QVA149 LAMA/LABA FDC DT with LAMA MT. They found a significantly reduced rate of moderate to severe exacerbations with the FDC DT therapy when compared to LAMA MT, for patients with GOLD stage III or IV COPD. Similarly, Buhl (2015) ${ }^{19}$ found a trend in improvement in moderate to severe exacerbations when comparing tiotropium/olodaterol FDC DT to either tiotropium or olodaterol MT. Our study found an incident rate ratio that would approximate 1 hospitalized exacerbation per 5.5 years in the MT cohort compared to 1 hospitalized exacerbation per
7 years in the DT cohort. The significance of a lower hospitalized exacerbation rate is that exacerbations are the major component of total COPD cost.

The results of the current study support the benefit of DT as demonstrated by lower mean COPD-related pharmacy and total costs PPPM for the DT versus MT cohort, and similar all-cause total costs PPPM for the 2 cohorts. Regression models showed lower mean allcause costs PPPM for the DT versus MT cohort. Other studies have had similar findings. Kozma $(2011)^{20}$ found higher COPD-related costs associated with LAMA or LABA MT versus LAMA/LABA DT. To have lower all cause costs in the DT group implies that COPD control is important for management of comorbidities and validates that the reduction in exacerbation frequency is meaningful. Meaningful reduction in cost and improved patient symptoms despite use of a more expensive fixed-dose inhaler is a worthwhile trade-off for the health care system. 


\section{Table 5. All-Cause and COPD-Related Health Care Resource Use and Costs per Patient per Month in the Follow-up}

\begin{tabular}{|l|c|c|c|c|c|c|}
\hline & \multicolumn{3}{|c|}{ All-Cause } & \multicolumn{3}{c|}{ COPD-Related } \\
\hline Follow-up & LAMA or & LAMALABA & P-value & LAMA or & LAMALABA & $P$-value \\
& LABA MT & FDC DT & & LABA MT & FDC DT & \\
& $(\mathrm{N}=1286)$ & $(\mathrm{N}=1286)$ & & $(\mathrm{N}=1286)$ & $(\mathrm{N}=1286)$ \\
\hline
\end{tabular}

Health Care Resource Utilization Counts PPPM, mean (SD)

\begin{tabular}{l|r|r|r|r|r|r}
\hline Ambulatory Visits & $2.32(1.76)$ & $2.34(1.96)$ & 0.788 & $0.75(0.77)$ & $0.75(0.81)$ & 0.881 \\
\hline Office Visits & $1.47(1.19)$ & $1.54(1.28)$ & 0.160 & $0.45(0.45)$ & $0.48(0.48)$ & 0.148 \\
\hline Outpatient Visits & $0.86(1.12)$ & $0.81(1.15)$ & 0.262 & $0.30(0.51)$ & $0.28(0.51)$ & 0.257 \\
\hline ED Visits & $0.11(0.24)$ & $0.10(0.23)$ & 0.486 & $0.06(0.20)$ & $0.05(0.15)$ & 0.116 \\
\hline Inpatient Stays & $0.04(0.08)$ & $0.04(0.08)$ & 0.232 & $0.04(0.08)$ & $0.03(0.08)$ & 0.092 \\
\hline Pharmacy Fills & $5.05(3.46)$ & $4.97(3.36)$ & 0.546 & $1.51(1.26)$ & $1.41(1.14)$ & 0.038 \\
\hline Tests and Procedures & - & - & - & $0.58(0.70)$ & $0.59(0.71)$ & 0.712 \\
\hline Health Care Costs (\$) PPPM, mean (SD) & & & & & \\
\hline Medical Costs & $1338.14(2459.70)$ & $1293.73(2332.14)$ & 0.639 & $823.23(1922.88)$ & $689.67(1628.10)$ & 0.057 \\
\hline Ambulatory & $590.12(1265.19)$ & $640.16(1352.47)$ & 0.333 & $203.02(478.80)$ & $200.94(438.50)$ & 0.908 \\
\hline Office Visits & $220.60(564.67)$ & $275.66(889.27)$ & 0.061 & $53.65(73.29)$ & $62.18(139.67)$ & 0.053 \\
\hline Outpatient Visits & $369.52(1098.76)$ & $364.50(916.51)$ & 0.900 & $149.37(458.09)$ & $138.75(389.28)$ & 0.527 \\
\hline ED Visits & $67.08(147.24)$ & $66.13(181.71)$ & 0.884 & $43.84(122.42)$ & $42.45(140.18)$ & 0.789 \\
\hline Inpatient Stays & $573.36(1691.69)$ & $464.62(1461.78)$ & 0.081 & $519.37(1610.61)$ & $402.29(1409.99)$ & 0.050 \\
\hline Other Medical Costs & $107.57(363.79)$ & $122.82(577.69)$ & 0.423 & $57.00(308.77)$ & $43.99(122.20)$ & 0.160 \\
\hline Pharmacy Costs & $717.30(879.66)$ & $686.82(1455.78)$ & 0.520 & $379.37(260.85)$ & $299.88(327.64)$ & $<0.001$ \\
\hline Total (medical + & $2055.44(2682.66)$ & $1980.55(2887.05)$ & 0.496 & $1202.60(1968.51)$ & $989.54(1688.97)$ & 0.003 \\
pharmacy) Costs & & & & & & \\
\hline
\end{tabular}

LAMA=long-acting muscarinic antagonist; LABA=long-acting beta2 agonist; MT=monotherapy; FDC=fixed-dose combination; $\mathrm{DT}=$ dual therapy; $\mathrm{COPD}=$ chronic obstructive pulmonary disease; $\mathrm{PPPM}=$ per patient per month; $\mathrm{SD}=\mathrm{standard}$ deviation; $\mathrm{ED}=$ emergency department

Laboratory and procedures performed during ambulatory visits, ED visits, and inpatient admissions were included in those costs. Laboratory and procedures performed at different locations, such as independent laboratories, were included in other costs. Costs were adjusted using the annual medical care component of the Consumer Price Index (CPI) to reflect inflation to year 2015. Costs are reported as PPPM.

\section{Limitations}

There are several limitations associated with this study. This was a real-world study, and patients initiating DT may have had more severe COPD than patients initiating $\mathrm{MT}$, which may have reduced the differences in outcomes seen between the 2 cohorts. Findings supporting more severe COPD in the DT cohort include a larger percentage of patients in the DT cohort having their index therapy prescribed by a pulmonologist and having higher numbers of baseline office visits, tests, and procedures compared to the MT cohort. It is possible that DT cohort patients seeing a pulmonologist could have received a different level of care than MT cohort patients seeing a general practitioner or internist.

One of the 3 definitions of an exacerbation was an ambulatory visit with a diagnosis in any position for COPD, and a procedure code for steroid and/or antibiotic administration during the visit, or a pharmacy claim for a steroid and/or antibiotic on the same day or within 10 days following the ambulatory visit. It is possible that patients may not have been experiencing an exacerbation at the time of their ambulatory visit but received a prescription in case they needed it for the future.

One advantage of real-world studies is that medication adherence can be measured, however a limitation is that there is no guarantee that a medication was taken after the prescription was filled, and in this study only the initial medication change was captured. The adherence findings from these cohorts showed that the DT cohort had a similar medication possession 
ratio but less persistence of therapy as measured by $\geq 1$ day without medication if the inhaler was used as prescribed. However, the MT patients augmented to triple therapy more often than DT patients and switched to an alternative therapy more often than the DT patients. The mixed results regarding medication adherence requires more focused study to define if medication cost, side effects, or efficacy is responsible for these differences. However, it is remarkable that the better outcomes recorded with the DT cohort occurred despite less medication use as measured by cost. A corollary observation is that the outcomes measured for the MT patients often occurred on medications that were augmented beyond MT, potentially reducing differences between groups.

Because LAMA/LABA FDC is a relatively new medication class, the numbers of patients available for analyses was small and required narrow windows surrounding inclusion and exclusion criteria. Whether relatively short wash-out periods surrounding medication use before the index date affected the differences between cohorts remains unknown.

It was not possible to obtain a spirometric diagnosis of COPD in this study design. However, in a related survey study ${ }^{5}$ that included a subset of the patients in this study, 95\% of patients in the MT cohort and 93\% in the DT cohort confirmed being told by a physician or health care provider that they have COPD, emphysema, or chronic bronchitis.

\section{Conclusions}

The DT cohort had lower rates of exacerbations leading to hospitalization, lower rates of switching and augmentation, and lower COPD-related pharmacy costs and total costs PPPM compared to the MT cohort. These data suggest greater clinical and economic benefits in patients with COPD taking DT versus MT. These findings also support earlier use of LAMA/ LABA FDC medications in all patients with COPD.

\section{Acknowledgments}

Author Contributions: All authors contributed to interpretation of data, drafting and revising the manuscript and gave final approval. Ms. Valery Walker and Ms. Maureen Carlyle also contributed to study design and collection of data. Dr. Junliang Tong also performed data analysis. Mr. Jonathan Kurlander also contributed to study design and performed data analysis.

We would like to thank Virginia M Rosen, PhD, an employee of Optum Inc, for her assistance in writing this manuscript.

\section{Declaration of Interest}

This study was funded by AstraZeneca Pharmaceuticals LP (Wilmington, Delaware). Optum, Inc., was contracted by AstraZeneca Pharmaceuticals LP to conduct this study and develop the manuscript.

Dr. Charlie Strange reports personal fees and nonfinancial support from AstraZeneca, CSA Medical, GlaxoSmithKline, and BTG, grants, personal fees, nonfinancial support from Pulmonx, personal fees from Uptake Medical, and grants from MatRx, all in COPD outside the submitted work. Mr. Jonathan Kurlander, Ms. Maureen Carlyle, Dr. Junliang Tong, and Ms. Valery Walker received support from AstraZeneca during the time that this study was conducted. Dr. Eric Wittbrodt reports that he is an employee of AstraZeneca. Dr. Lauren A. Millette reports that she was an employee of AstraZeneca during the time that this study was conducted and is currently an employee of Genentech. The authors report no other conflicts of interest in this work. 


\section{References}

1. Kochanek KD, Murphy S, Xu J, Arias E. Mortality in the United States, 2016. NCHS Data Brief. 2017;(293):1-8.

2. Global Initiative for Chronic Obstructive Lung Disease (GOLD). The Global Strategy for the Diagnosis, Management and Prevention of COPD, 2017. GOLD website. http://goldcopd.org. Published 2017. Accessed June 2019.

3. Ford ES, Murphy LB, Khavjou W, et al. Total and state-specific medical and absenteeism costs of COPD among adults aged $\geq$ 18 years in the United States for 2010 and projections through 2020. Chest. 2015;147(1):31-45.

doi: https://doi.org/10.1378/chest.14-0972

4. Dalal AA, Patel J, D'Souza A, et al. Impact of COPD exacerbation frequency on costs for a mnaged care population. J Manag Care Spec Pharm. 2015;21(7):575-583.

doi: https://doi.org/10.18553/jmcp.2015.21.7.575

5. Strange C, Walker V, DePietro $\mathrm{M}$, et al. Patient-reported outcomes of dual bronchodilator fixed-dose combination versus bronchodilator monotherapy in individuals with COPD. Int $J$ Chron Obstruct Pulmon Dis. 2019. In press.

6. U.S. Department of Labor, Bureau of Labor Statistics. Consumer Price Index: medical care. Series ID: CUUR0000SAM. U.S. Department of Labor website. http://data.bls.gov/cgi-bin/survey most?su . Published 2012. Accessed June 2019.

7. Austin PC. Balance diagnostics for comparing the distribution of baseline covariates between treatment groups in propensityscore matched samples. Stat Med. 2009; 28(25):3083-3107. doi: https://doi.org/10.1002/sim.3697

8. Quan H, Li B, Couris CM, et al. Updating and validating the Charlson comorbidity index and score for risk adjustment in hospital discharge abstracts using data from 6 countries. Am J Epidemiol. 2011;173(6):676-682.

doi: https://doi.org/10.1093/aje/kwq433

9. Wu EQ, Birnbaum HG, Cifaldi M, et al. Development of a COPD severity score. Curr Med Res Opin. 2006; 22(9):1679-1687. doi: https://doi.org/10.1185/030079906X115621

10. Agency for Healthcare Research and Quality (AHRQ). Healthcare Cost and Utilization Project comorbidity software. AHRQ website. www.hcup-us.ahrq.gov/toolssoftware/comorbidity /comorbidity.jsp. Published 2015. Accessed June 2019.

11. Higgins BG, Powell RM, Cooper S, Tattersfield AE. Effect of salbutamol and ipratropium bromide on airway calibre and bronchial reactivity in asthma and chronic bronchitis. Eur Respir J. 1991;4(4):415-420.

12. Beeh KM, Burgel PR, Franssen FME, et al. How do dual longacting bronchodilators prevent exacerbations of chronic obstructive pulmonary disease? Am J Respir Crit Care Med. 2017;196(2):139-149.

doi: https://doi.org/10.1164/rccm.201609-1794CI
13. Rodrigo GJ, Price D, Anzueto A, et al. LABA/LAMA combinations versus LAMA monotherapy or LABA/ICS in COPD: a systematic review and meta-analysis. Int $J$ Chron Obstruct Pulmon Dis. 2017;12:907-922. doi: https://doi.org/10.2147/COPD.S130482

14. Calzetta L, Ora J, Cavalli F, et al. Impact of LABA/LAMA combination on exercise endurance and lung hyperinflation in COPD: a pair-wise and network meta-analysis. Respir Med. 2017;129:189-198.

doi: https://doi.org/10.1016/j.rmed.2017.06.020

15. Diette GB, Dalal AA, D'Souza AO, et al. Treatment patterns of chronic obstructive pulmonary disease in employed adults in the United States. Int J Chron Obstruct Pulmon Dis.2015;10:415422. doi: https://doi.org/10.2147/COPD.S75034

16. Make B, Dutro MP, Paulose-Ram R, et al. Undertreatment of COPD: a retrospective analysis of US managed care and Medicare patients. Int J Chron Obstruct Pulmon Dis. 2012;7:1-9. doi: https://doi.org/10.2147/COPD.S27032

17. Tran M, Xiang P, Rascati KL, et al. Predictors of appropriate pharmacotherapy management of copd exacerbations and impact on 6-Month readmission. Manag Care Spec Pharm. 2016;22(10):1186-1193.

doi: https://doi.org/10.18553/jmcp.2016.22.10.1186

18. Wedzicha JA, Decramer M, Ficker JH, et al. Analysis of chronic obstructive pulmonary disease exacerbations with the dual bronchodilator QVA149 compared with glycopyrronium and tiotropium (SPARK): a randomised, double-blind, parallel-group study. Lancet Respir Med. 2013;1(3):199-209.

doi: https://doi.org/10.1016/S2213-2600(13)70052-3

19. Buhl R, Maltais F, Abrahams R, et al. Tiotropium and olodaterol fixed-dose combination versus mono-components in COPD (GOLD 2-4). Eur Respir J. 2015;45(4):969-979. doi: https://doi.org/10.1183/09031936.00136014

20. Kozma CM, Paris AL, Plauschinat CA, et al. Comparison of resource use by COPD patients on inhaled therapies with longacting bronchodilators: a database study. BMC Pulm Med. 2011 11:61. doi: https://doi.org/10.1186/1471-2466-11-61 